\title{
Dilation Kinetics of Glassy, Aromatic Polyimides Induced by Carbon Dioxide Sorption
}

\author{
M. WESSLING,* I. HUISMAN, Th. v. d. BOOMGAARD, and C. A. SMOLDERS \\ University of Twente, Chemical Technology, P.O. Box 217, 7500 AE Enschede, The Netherlands
}

\begin{abstract}
SYNOPSIS
Over the past years, the equilibrium sorption of gases in polymers has been intensively studied. Mostly, glassy polymers were investigated because of their excellent selective mass transport properties. This work does not focus on the equilibrium sorption but on the kinetics to reach the equilibrium. We developed a new experimental method measuring the sorption-induced dilation kinetics of a polymer film. Carbon dioxide and glassy, aromatic polyimides were chosen as model systems. Low-pressure experiments demonstrate that the measured dilation kinetics represent the sorption kinetics. A significant delay between the sorption and dilation kinetics is based on the fact that dilation kinetics occurs simultaneously with the concentration increase in the center of the polymer film. High-pressure experiments reveal significant differences in dilation kinetics compared to low-pressure experiments. Generally, three regimes can be distinguished in the dilation kinetics: a first, fast volume increase followed by two much slower regimes of volume increase. The magnitude of fast and slow dilation kinetics strongly depends on the swelling history of the polymer sample. The results of the experiments are analyzed in the light of a model relating the fast dilation kinetics to a reversible "Fickian" dilation and the slower dilation kinetics to an irreversible, relaxational dilation. (c) 1995 John Wiley \& Sons, Inc.
\end{abstract}

Keywords: sorption • dilation • carbon dioxide $\bullet$ polyimides

\section{INTRODUCTION}

The success of industrial gas separation using polymeric membranes ${ }^{1}$ and the widespread application of polymer films as barrier materials, ${ }^{2}$ has boosted the experimental and theoretical research on mass transport through polymer films. In particular, the success of glassy polymers in gas separation membranes caused an intensive search for tailor-made polymers exhibiting improved selective mass transport properties. ${ }^{1-6}$ It is generally accepted that mass transport through polymer films can be described by the solution-diffusion model: penetrant molecules dissolve in the polymer matrix at the side of the membrane with the highest chemical potential and diffuse through the polymer matrix into the direction of the lower chemical potential. Over the last thirty years, many theoretical models have been developed

\footnotetext{
* To whom correspondence should be addressed.

Journal of Polymer Science: Part B: Polymer Physics, Vol. 33, 1371-1384 (1995) (C) 1995 John Wiley \& Sons, Inc. CCC 0887-6266/95/091371-14
}

describing the sorption process into the polymer. Generally, the models describe the sorption equilibrium, rather than the dynamics to reach this equilibrium.

The sorption of penetrant molecules into a rubbery polymer is well described by the Flory-Huggins equation. ${ }^{7}$ However, for the sorption of penetrant molecules into glassy polymers, a number of different models have been developed. The most widely used model is the dual-mode sorption model..$^{8-10}$ Other models describing sorption into glassy polymers are: (a) the gas-polymer matrix model of Raucher and Sefcik, ${ }^{11}$ (b) the thermodynamic model of Vrentas and Vrentas ${ }^{12}$ accounting for the penetrant-induced glass transition temperature depression, (c) the elastic strain corrected lattice model of Bitter, ${ }^{13}$ (d) the thermodynamic model of Lipscomb ${ }^{14}$ accounting for elastic deformation, (e) the glassy polymer lattice sorption model of Barbari and Conforti, ${ }^{15}$ (f) the affinity distribution model of Weiss et al., ${ }^{16}$ and (g) the site energy distribution model of Kirchheim. ${ }^{17}$ With this study we do not attempt to verify one or 
the other model describing the sorption equilibrium. In contrary, the present work focuses on the sorption-induced dilation kinetics.

The information on the dilation kinetics may be particularly important in membrane applications where a glassy polymer is exposed to a gas or liquid phase with a high affinity to the polymer. Two phenomena, generally referred to as relaxation or plasticization phenomena, can be observed:

- The permeability strongly depends on the history of the membrane ${ }^{18,19}$

- The permeability is not constant but changes continuously in time ${ }^{20-23}$

The purpose of the study is to elucidate relaxational mass transport phenomena occurring during mass transport into glassy polymers. The volume change of the polymer/penetrant system during the sorption process, measured with a newly developed experimental technique, is taken as the experimental measure to quantify these relaxation phenomena.

\section{EXPERIMENTAL}

\section{Polymer Synthesis and Characterization}

For the characterization of dilation kinetics, two aromatic polyimides based on the dianhydride 6FDA [4,4'-(2,2,2-trifluoro methyl)ethylidene]bis[1,isobenzo-furan-dione]) were synthesized following the procedure previously described. ${ }^{24}$ The completion of the imidization reaction was observed by ${ }^{1} \mathrm{H}$ NMR and IR spectroscopy. IR spectra showed distinct absorption at wavelengths of 1785 and 1720 $\mathrm{cm}^{-1}$ for the imide bonds whereas no absorption was observed for the amide bonds at 2865-3100, 1655, and $1540 \mathrm{~cm}^{-1}$. Homogeneous films were prepared by casting a 10-15 wt \% solution of the synthesized polyimide in dimethyl-acetamid DMAc on a glass plate, which was dried for two days under a stream of nitrogen. After solvent evaporation, the films were released from the glass plate by immersion in a water bath. Samples were then dried for at least 2 weeks in a vacuum oven at $150^{\circ} \mathrm{C}$ to remove the residual solvent. After drying, no characteristic solvent peaks could be found in the IR spectra. Heating above the glass transition temperature is often used to remove any solvent molecules not detected by infrared spectroscopy. However, we did not use this procedure because heat treatment may transform samples with smooth surfaces and geometry into "wavy" samples. Dilation experiments with a distorted sample would be difficult; therefore, to maintain a uniform drying procedure for the sorption samples and the dilation samples, all samples were prepared as described above.

The polymers synthesized are listed in Table I. The glass transition temperature $T_{g}$ was obtained by DSC measurements performed on a Perkin-Elmer DSC-4 calorimeter at a heating rate of $20^{\circ} \mathrm{C} /$ min. Polymer densities were measured using a setup where the weight of a polymer sample is measured in air and in a liquid (water or iso-octane). The difference in the two weights is an accurate measure of the polymer density. ${ }^{22}$ The data for the glass transition temperature and densities listed in Table I agree well with the data reported elsewhere. ${ }^{25}$

\section{Dilation}

A new, automated experimental technique has been developed to determine the swelling of a polymer induced by the sorbed gas. A pressure cell with two glass windows contains a hanging polymer strip, as shown in Figure 1 . The polymer strip, about $10 \mathrm{~cm}$ long, is fixed at the top of the cell and placed on a porous clamping fixture, $9 \mathrm{~cm}$ long. This construction guarantees a stable position for the polymer sample as well as contact of the total sample surface with the gas phase. Upon gas uptake, the polymer swells isotropically as described in the literature. ${ }^{26}$ The movement of the end of the strip toward the bottom of the cell, which is visible through the windows, is a measure of the sorption-induced dilation. The pressure and the temperature in the cell were continuously controlled. In the past, as reported in literature, the displacement of the end of the strip was observed with a cathetometer ${ }^{27}$ or a video camera. ${ }^{26}$ These techniques, however, make the measurement of the kinetics of volume increase very difficult. ${ }^{27}$ For this study, the observation of the displacement of the end of the film strip was automated by using a line scan camera, allowing more detailed measurement of dilation kinetics.

An external light source illuminates the cell so that a small, opaque mark at the end of the polymer strip is easily visible. This mark is the optical indication of the end of the strip. For the accuracy of the experiments, it did not matter whether this mark was a gold strip sputtered on the polymer surface, a small tape strip, or a small metal strip clamped to the end of the sample. The line-scan camera, consisting of a line of 2064 light-sensitive diodes, measures the light intensity with respect to the local position. Each diode measured the light intensity on a scale from 0 to 64 . The resolution of the line-scan 
camera is $5.2 \mu \mathrm{m}$. A "photo" or line scan of the end of a polymer strip hanging in the cell is shown in Figure 2. At the opaque mark, the measured light intensity drops to zero. A sudden change in light intensity cannot be observed because of refraction effects at the edge of the mark. However, the width of the transition region from high to the low intensity did not change if the pressure or temperature was changed. Various mathematical techniques to transform this transition region into a single value, the transition point, have been investigated. The most accurate and reliable definition of the end of the strip, the transition point, is given by the diode which shows the first time an intensity of zero coming from diodes with higher light intensities. This transition point can then be followed over time as a function of the pressure and temperature. The automation of the experimental procedure enables the determination of the transition point every $9 \mathrm{~s}$ and makes it possible to determine accurately the dilation kinetics.

\section{Sorption Kinetics}

To determine the sorption kinetics, a set-up was used that is based on a pressure-decay method. ${ }^{28,29}$ The set-up consists of two cells of which one contains the polymer sample and the second one is an empty reference cell. Both cells are placed in a thermostated liquid bath. Additionally, the whole setup is placed in thermostated air bath having the same temperature as the liquid bath. The mass uptake by the polymer is measured as a minute pressure difference decay between the cells. The final pressure decay in a sorption kinetics experiment is

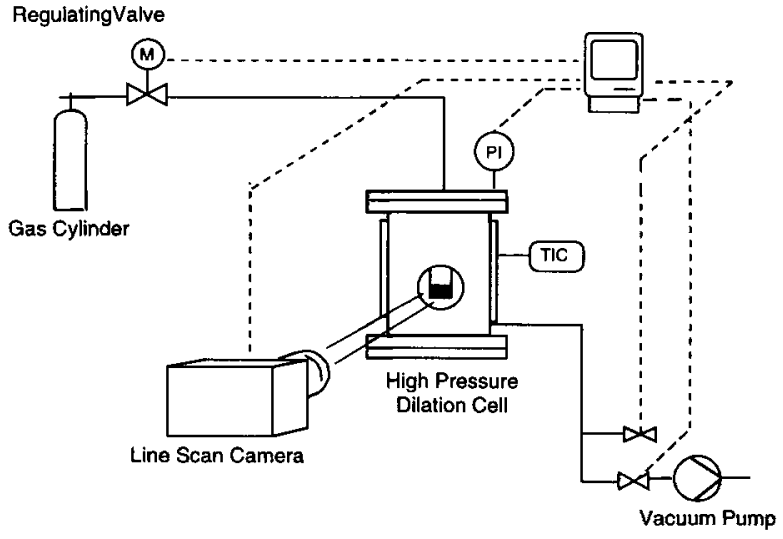

Figure 1. High pressure dilation set-up.

small $(<5 \%)$ compared to the absolute pressure in the system.

\section{EXPERIMENTAL RESULTS}

To demonstrate that the measured dilation kinetics is related to sorption kinetics, low-pressure sorption and dilation kinetics experiments up to 3 bar will be compared in the first part of this section. In the second part of this section, high-pressure dilation experiments up to 36 bar will be described. There, the focus will be on the dilation kinetics as a function of the polymer history.

\section{Low Pressure Dilation}

Initial screening experiments determining the sorption and dilation kinetics were carried out with films

Table I. Chemical Structure and Physical Properties of Aromatic Polyimides Used for Dilation Experiments

Polymer Name
$\begin{gathered}\text { Glass Transition } \\ \text { Temperature } \\ \left({ }^{\circ} \mathrm{C}\right)\end{gathered}$




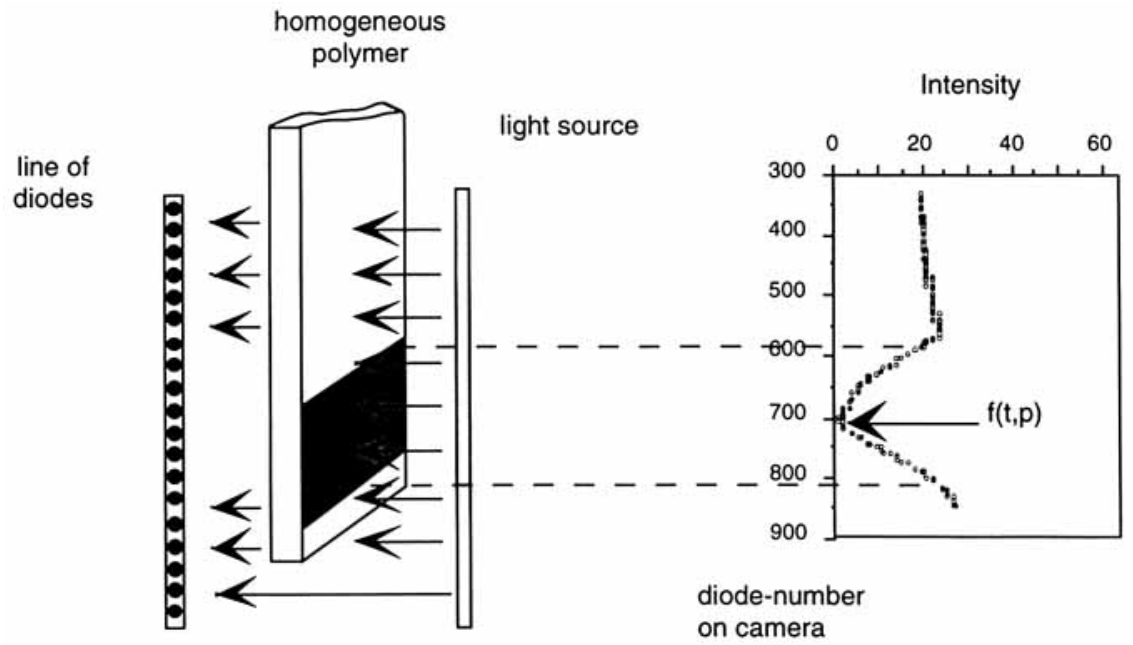

Figure 2. Measurement principle of line-scan camera used to determine dilation kinetics of a polymer strip hanging inside a high pressure cell.

cast from the commercial polyimide PIXU218 (Ciba Geigy). Comparing the dilation and sorption kinetics of $\mathrm{CO}_{2}$ in the polyimide PIXU 218 for a pressure step from 0 to 3 bar, a peculiar phenomenon can be observed as shown in Figure 3. (The time axis in Figure 3 is transformed into the square-root-of-time axis as usually done for the analysis of mass transport kinetics.) Clearly, there is a considerable delay between the carbon dioxide uptake and the strip length increase. Three possible reasons exist:

1. The pressure in the dilation cell increases gradually, whereas the pressure in the sorption setup increases instantly, as shown in Figure 4. The pressure in the dilation cell first increases with a pressure step from 0 to 0.8 bar and then increases continuously to the pressure level required. The pressure in the dilation cell was regulated in this manner because a sudden pressurization from vacuum

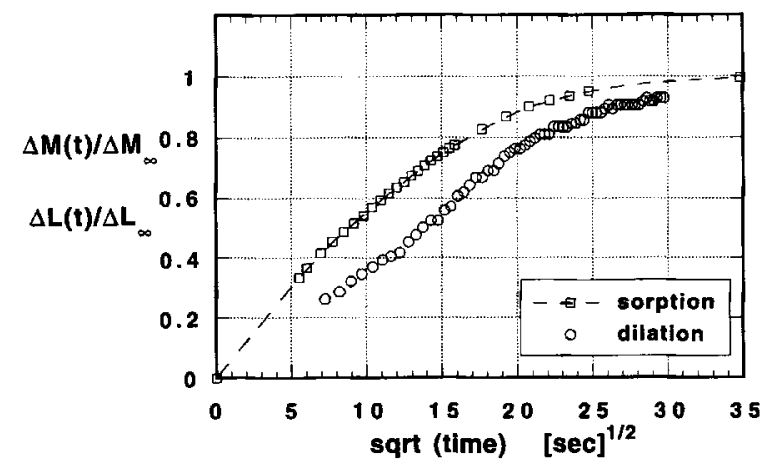

Figure 3. Comparison of the sorption and dilation kinetics of $\mathrm{CO}_{2}$ sorption in PIXU218 at $T=25^{\circ} \mathrm{C}$. to 3 bar may have caused the sample to change its position upon pressurization. However, from Figure 4 it also can be concluded that mass transport into the film occurs at the very beginning of the experiments because of the initial pressure step from 0 to 0.8 bar. The continuous increase in pressure from 0.8 to 3 bars, however, represents a timedependent boundary condition in the mathematical description of the mass transport kinetics.

2. Glassy polymers contain unrelaxed free volume which can accommodate penetrant molecules without dilation of the matrix. ${ }^{30}$ However, the sorption and dilation isotherms and the cross-plot thereof, as shown in Figure 5, contradict this interpretation. Figure 5c shows the volume increase of the polymer sample as a function of penetrant concentration. (The volume increase can be calculated from the dilation experiment assuming isotropic swelling: see also eqs. (6) and (7).)

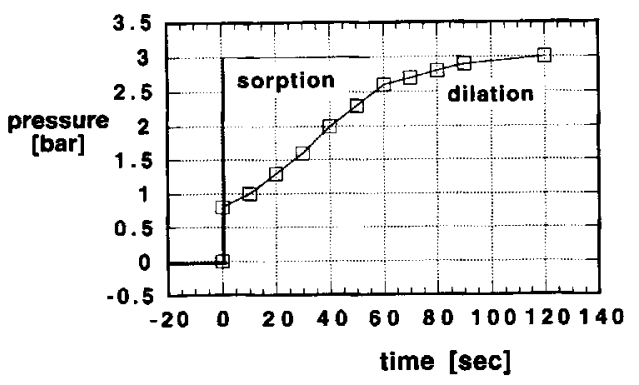

Figure 4. Comparison of the pressurization step for the sorption and dilation set-up. 
(a) concentration $\left[\mathrm{cm}^{3}(\mathrm{STP}) / \mathrm{cm}^{3}\right.$ polym.]

(b)

(c)

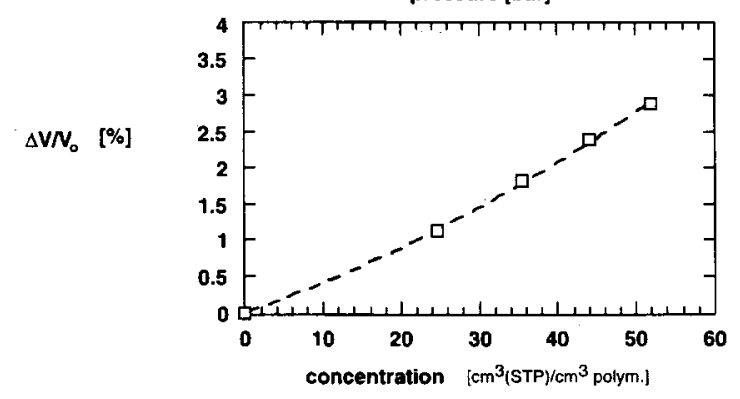

Figure 5. (a) Sorption and (b) dilation isotherm for $\mathrm{CO}_{2}$ sorption in PIXU218 at $T=25^{\circ} \mathrm{C}$; (c) cross-plot of sorption and dilation isotherm.

The volume increases almost linearly with the penetrant concentration indicating that any molecule entering the polymer matrix swells the polymer. Therefore, the delay in the dilation kinetics is not related to the unrelaxed free volume of the glassy polymer.

3. The observed delay time in dilation must be considered a mass transfer problem and may be closely related to the film thickness of the polymer sample. Figure 6 illustrates the problem of comparing sorption and dilation kinetics. Penetrant molecules diffuse into the depth of the polymer film until the concentration is equal over the whole film thickness. Dilation, however, is a time-dependent phenomenon in the length direction of the film. It is not clear whether dilation can already be observed at $t=0$, when the first molecules are sorbed in the surface layer of the film, or whether dilation starts when the first molecules have reached the middle of the strip. The latter is suggested by the delay between

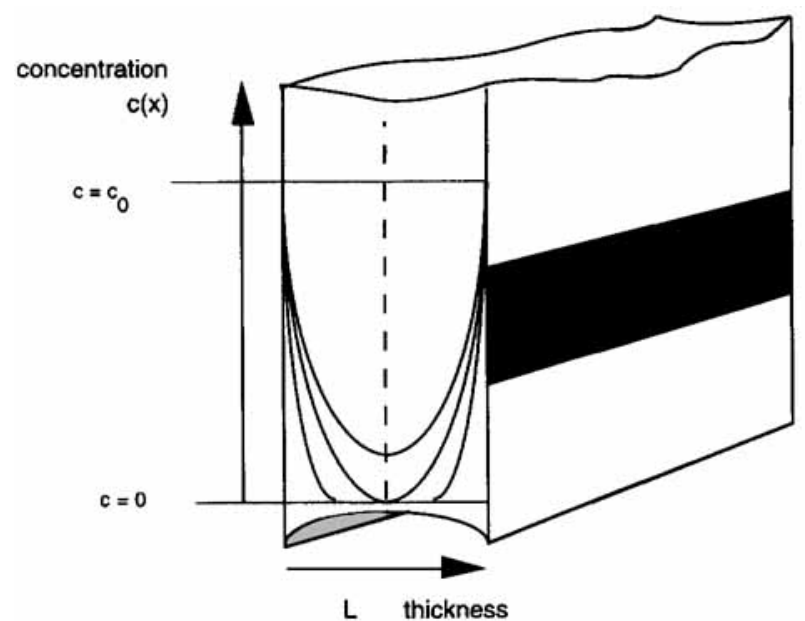

Figure 6. Schematic representation of concentration profiles in polymer film as time proceeds in a dilation kinetic experiment.

sorption and dilation and has been reported for some special cases of liquid sorption into glassy polymers. ${ }^{31}$

To resolve this open question, further experiments were carried out with $\mathrm{CO}_{2}$ and the polyimide 6FDA-DAF. The sorption and dilation kinetics was measured at $25^{\circ} \mathrm{C}$ for a pressure step from 0 to 3 bar. The sorption kinetics was fitted using eq. (1). Fitted and experimental data agreed very well and, for clarity purposes only, the fitted sorption kinetics is shown as a solid line in Figure 7. The dilation kinetics of a $122-\mu \mathrm{m}$ thick sample is also plotted in Figure 7. (The measured dilation kinetics shows incremental steps because the line-scan camera has a minimum resolution of $5.2 \mu \mathrm{m}$.) Both the mass uptake and the dilation kinetics are compared with the

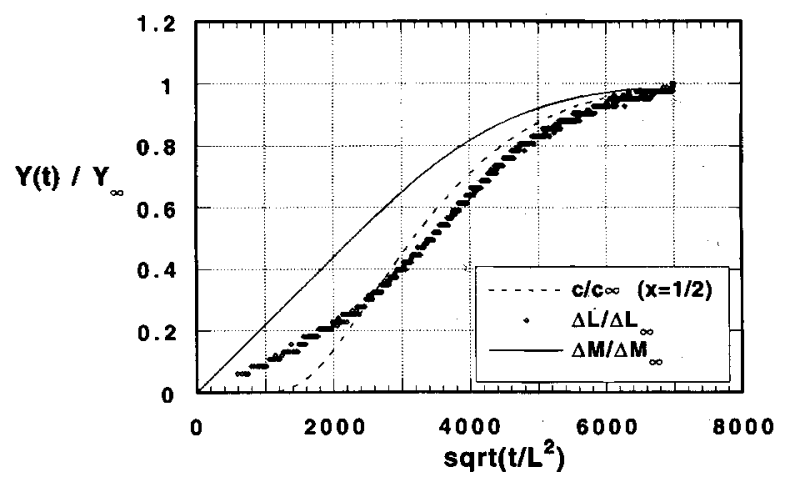

Figure 7. Comparison of calculated total mass uptake and the concentration in the middle of the polymer film with experimentally determined dilation kinetics for the system 6 FDA-DAF at $T=25^{\circ} \mathrm{C}$ and a film thickness of $122 \mu \mathrm{m}$. 
calculated relative concentration increase in the middle $(x=L / 2)$ of the polymer strip ${ }^{32}$ as given by eq. (2).

$$
\begin{aligned}
& \frac{M(t)}{M_{\infty}}=1-\frac{8}{\pi^{2}} \sum_{m=0}^{\infty} \frac{1}{(2 m+1)^{2}} \\
& \times \exp \left\{-\frac{D(2 m+1)^{2} \pi^{2} t}{L^{2}}\right\} \\
& \frac{c(t)}{c_{\infty}}\left(x=\frac{L}{2}\right)=1 \\
& -\frac{4}{\pi} \sum_{m=0}^{\infty} \frac{(-1)^{m}}{(2 m+1)} \exp \left\{\frac{D(2 m+1)^{2} \pi^{2} t}{L^{2}}\right\}
\end{aligned}
$$

From a certain inflection point in the graph, for $\Delta L(t) / \Delta L_{\infty}>0.35$, the length of the polymer strip changes simultaneously with the concentration in the middle of the film but not with the total mass uptake. This observation supports the third explanation given for the delay in dilation. The delay between the dilation curve and the concentration in the center of the film, however, may be caused by limitations in the pressure regulation as described in the first argument.

A significant deviation of the dilation curve from the concentration curve is seen in Figure 6 for values below $\Delta L(t) / \Delta L_{\infty}<0.35$. Here, the dilation increases less markedly than the total mass uptake but more quickly than the calculated concentration in the middle of the film. To elucidate this problem, similar experiments were carried out with $122-\mu \mathrm{m}$ sample at different temperatures and with the 46 $\mu \mathrm{m}$ sample at $25^{\circ} \mathrm{C}$. Figure 8 compares the dilation kinetics of the thick sample at three different temperatures 25,35 , and $45^{\circ} \mathrm{C}$ with dilation kinetics of the thin polymer sample. Again, for clarity the experimental data are represented by lines. The circles in Figure 8 indicate the bending points for the thick sample. The thin sample does not show any bending point. It even appears that the thin sample does not dilate at all for times smaller than 100 seconds. However, the absence of the dilation is only apparent. In fact, we expect the bending point for the thin sample to be at lower relative dilation and smaller times. The measurements carried out with the thick sample at increasing temperatures support this interpretation: increasing the temperature increases the diffusion coefficient which has the same qualitative effect as decreasing the membrane thickness causing the bending point to move toward smaller relative dilation at smaller times. Apparently, the measured dilation is caused by a superposition of

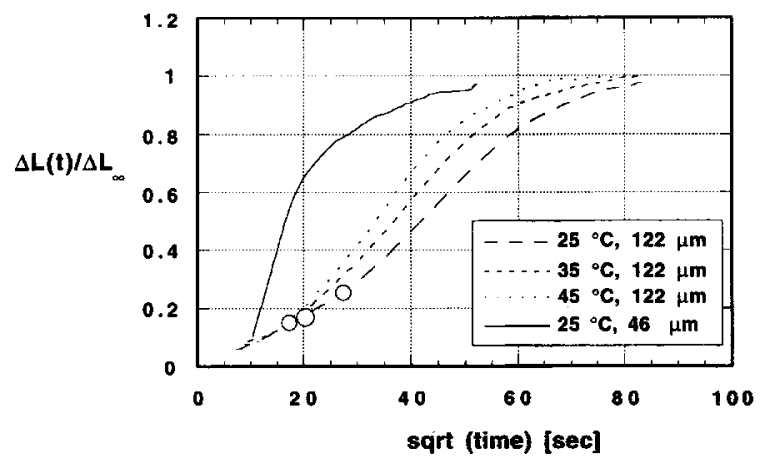

Figure 8. Dilation kinetics for the system 6FDA-DAF/ $\mathrm{CO}_{2}$ at different temperatures and different sample thicknesses; circles show bending points in curve.

swelling in the thickness and length direction of the film. The larger the diffusion coefficient or the less the polymer film thickness, the smaller is the contribution of the complex swelling behavior to the dilation kinetics.

We summarize the dilation experiments at low pressures:

1. For times smaller than the time at which the bending point occurs, the observed dilation is a complex combination of swelling in the thickness and in the length direction of the film

2. From the bending point on, the polymer film dilates simultaneously with the concentration increase in the middle of the film. The observed dilation signal is therefore an accurate measure for mass transport

\section{High Pressure Dilation}

The foregoing section described the dilation kinetics at low carbon dioxide pressures. The experiments were carried out in a pressure step from 0 to 3 bar. In this section, we describe two different kinds of experiments at elevated pressures up to 40 bars:

- Dilation experiments with consecutive pressure steps of 3 bars during dilation (sorption) and pressure steps of 10 bars during consolidation (desorption)

- Dilation experiments with integral pressure steps from 0 to $20 \mathrm{bar}$

The experiments were carried out with both polyimides: 6FDA-DAF and 6FDA-MDA. The obtained experimental results will be described below for each 


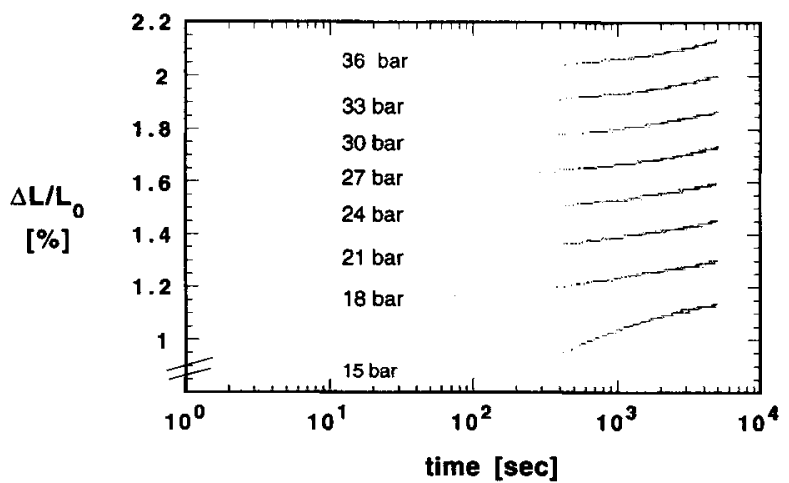

Figure 9. Consecutive dilation experiments at pressures $p \geq 15$ bar for the system 6 FDA-DAF $/ \mathrm{CO}_{2}$ at $T=25^{\circ} \mathrm{C}$.

polymer separately and discussed in a separate section.

\section{Dilation Experiments Carried Out With 6FDA-DAF}

Dilation experiments at consecutive pressure steps were carried out with the polyimide 6FDA-DAF. A pressure range from 0 to 36 bar was evaluated in pressure steps of 3 bars. At each pressure, the experiment lasted $5000 \mathrm{~s}$. With a photo frequency of 1 photo per $9 \mathrm{~s}$, around 550 data points were collected at each pressure.

For pressures up to 15 bar, dilation reached equilibrium within the experimentation time. Dilation at higher pressures, however, does not reach equilibrium as shown in Figure 9. The fact that equilibrium is not reached at high pressures is unexpected. Since the diffusion coefficients increase with increasing concentration ${ }^{23,33}$ (increasing pressure), one anticipates a quicker approach to the equilibrium dilation.

Figure 10 shows the dilation kinetic obtained when the pressure is decreased in a first pressure step of 5 bar and three consecutive steps of 10 bar each from 36 bar to 1 bar. The polymer film consolidates since carbon dioxide desorbs from the polymer sample. However, the consolidation kinetics does not follow the dilation kinetics. A pressure decrease from 36 to 31 bar practically does not show any consolidation whereas a pressure increase from 30 to 36 bar resulted in a relative dilation increase of 0.25 . A pressure decrease from 31 to 21 bar shows significantly less consolidation than the pressure decrease from 11 to 1 bar. Evacuation at $p=0$ bar for $7000 \mathrm{~s}$ results in a residual dilation value of $0.19 \%$ (not shown) as a pseudo-equilibrium; further evacuation for $72 \mathrm{~h}$ decreases this residual dilation down to $0.05 \%$.

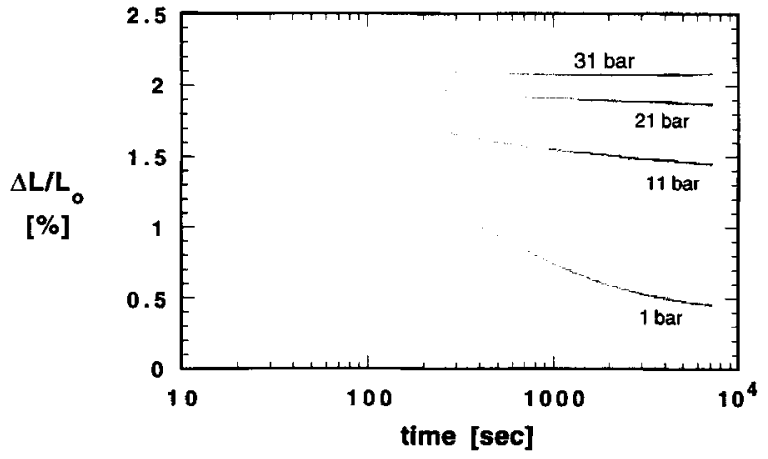

Figure 10. Consolidation kinetics for the desorption of $\mathrm{CO}_{2}$ from 6FDA-DAF at $T=25^{\circ} \mathrm{C}$ in pressure steps of 10 bar.

One can transform the dilation and consolidation kinetics experiments into a dilation and consolidation isotherm. For experiments below 15 bar pressure, the equilibrium dilation is experimentally determined; for the experiments at pressures larger than 15 bar and for consolidation experiments, the last data point of the kinetics experiment may be assumed as a pseudo-equilibrium. The result of this analysis is shown in Figure 11. A significant hysteresis between dilation and consolidation can be observed. This phenomenon has also been measured for the polyimide PIXU 218 but data are not shown here. Several other authors already mentioned this dilation and consolidation hysteresis ${ }^{19,34}$ and it can be considered as a general behavior of glassy polymers.

\section{Dilation Experiments Carried Out With 6FDA-DAF}

Similar dilation experiments with $\mathrm{CO}_{2}$ as a sorbing gas have been carried out with the polyimide 6FDAMDA at $T=35^{\circ} \mathrm{C}$. Dilation kinetics were measured

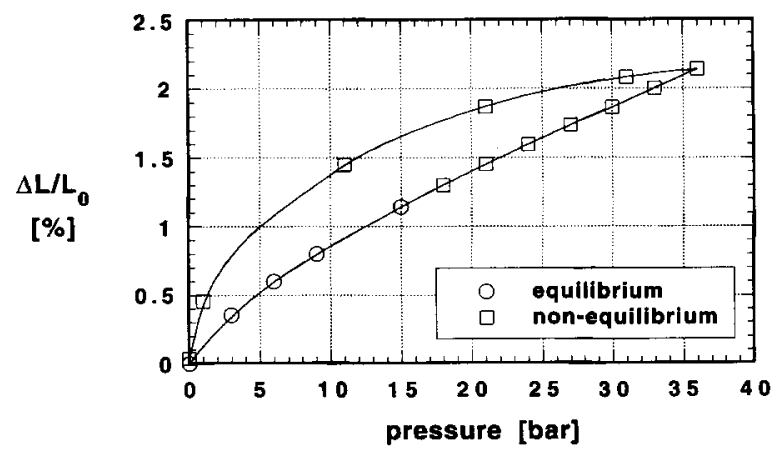

Figure 11. Dilation and consolidation isotherms for the system of $6 \mathrm{FDA}-\mathrm{DAF} / \mathrm{CO}_{2}$ at $T=25^{\circ} \mathrm{C}$; equilibrium times as $5000 \mathrm{~s}$ for dilation and 8000 seconds for consolidation. 
for incrementally increasing pressure steps of 3 bar as well as for integral pressure steps from 0 to 20 bar, for example. Desorption experiments were not carried out. A typical result, obtained for such an integral pressure step, is shown in Figure 12. The relative length dilation is plotted versus a logarithmic time axis and the time is normalized for the square of the film thickness. The latter is preferably done to make the product of diffusion coefficient and the ratio of time and squared film thickness dimensionless so that experiments with samples having different thicknesses can be compared. The choice of a logarithmic time scale allows to distinguishing dilation at short and long experimentation times. Dilation was measured from relative dilation values larger than about 0.7 . For smaller times no measurement could be taken because of pressure control mechanism. However, a pressure close to the final value was very quickly reached but the time to fine-tune the final pressure took as long as the pressure increase.

In Figure 12, at least three regimes can be distinguished. An initial, very fast: a second, slower: and a third steady state equilibrium regime. Similar dynamic responses have been observed by Berens and Hopfenberg ${ }^{35}$ for the weight gain in a sorption experiment of poly (vinylchloride) with different organic vapors such as ethanol, acetone, and vinylchloride. In the following we will adopt their terminology to describe the experimental findings. The first regime $\left(\Delta L(t) / \Delta L_{\infty}<0.78\right)$ stands for the sorption process where the length increase of the sample follows the concentration increase in the middle of the film. We will refer to this behavior as "Fickian" and the relative amount of Fickian dilation will be called $\phi_{F}$. The Fickian dilation is followed by at least one regime which is considerably slower. Such a regime will be called "relaxational." The relative extent of these relaxation dilations will be called $\phi_{R}$. The last regime is actually the dilation equilibrium.

Berens and Hopfenburg ${ }^{35}$ modeled their experiments phenomenologically assuming that the total mass uptake consists of ordinary Fickian mass uptake and secondly of mass uptake due to relaxation.

$$
M(t)=M(t)_{F}+M(t)_{R}
$$

The dilation experiments at low pressures, described in the previous section, showed that the measured dilation represents mass uptake. Hence, Berens' approach can also be transformed to dilation experiments:

$$
V(t)=V(t)_{F}+V(t)_{R}
$$

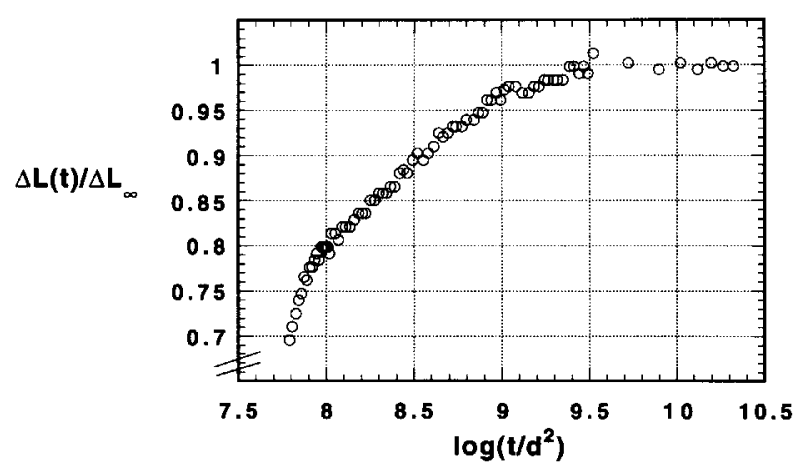

Figure 12. Dilation kinetics for the sorption of $\mathrm{CO}_{2}$ in 6FDA-MDA at $T=35^{\circ} \mathrm{C}$ for an integral pressure step from $p=0$ to $p=20 \mathrm{bar}$.

Assuming isotropic swelling with $\varepsilon=\Delta L / L_{0}$

$$
V=V_{0}(1+\varepsilon)^{3}
$$

and neglecting second order and third order terms at low relative dilation, eq. (6) can be transformed into

$$
V=V_{0}(1+3 \varepsilon)
$$

This proportionality means that instead of investigating the volume increase it is also valid to investigate the length increase without losing any significant information. This will be done in the following: normalizing the absolute length increase at a time $t$ by the equilibrium length increase, eq. (5) can be rewritten as

$$
\frac{\Delta L(t)}{\Delta L_{\infty}}=\frac{\Delta L(t)_{F}}{\Delta L_{\infty}}+\frac{\Delta L(t)_{R}}{\Delta L_{\infty}}=\phi_{F}(t)+\phi_{R}(t)
$$

Adapting the mathematical treatment of viscoelastic properties of polymers, the relative relaxational length increase can be written as a sum of phenomenological functions representing a distribution of first order processes with rate constants $k_{i}=1 / \tau_{i}$ where $\tau_{i}$ is the relaxation time.

$$
\phi_{R}(t)=\sum_{n=1}^{\infty} \phi_{R, n}\left(1-\exp \left\{-\frac{t}{\tau_{R n}}\right\}\right)
$$

Figure 13 shows an example of the mathematical treatment described above. The total relative dilation is equal to the sum of the Fickian and the relaxational dilation regimes. In Figure 13a, the relative Fickian dilation and two relaxational dilation kinetics are shown separately. The relaxation times 

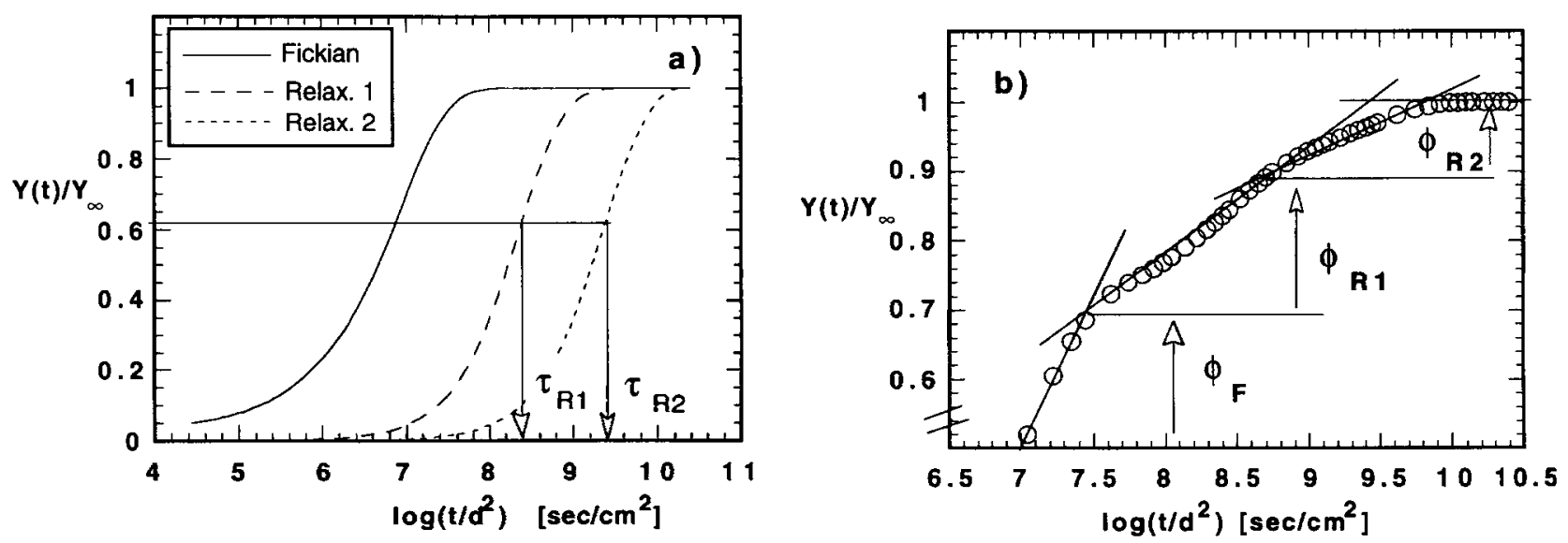

Figure 13. (a) Fickian length increase and two relaxational functions $Y(t) / Y_{\infty}=1$ $-\exp \{-t / \tau\}$ plotted versus the logarithmic, membrane thickness normalized time for different (membrane thickness normalized) relaxation times $\tau_{R 1}=2.5 E 8$ and $\tau_{R 2}=2.5 E 9$ $\mathrm{s} / \mathrm{cm} 2 d=60 \mu \mathrm{m}$ ); (b) summation of percentages of the three functions according to the above described procedure $\left(\phi_{f}=0.7, \phi_{R 1}=0.2, \phi_{R 2}=0.1\right)$; data points are calculated, solid lines have no mathematical meaning but solely point out the three regimes.

for the latter two can be determined assuming that $t=\tau_{R i}$; the corresponding $y$-value equals

$$
Y(t) / Y_{\infty}=1-\exp \{-1\}=0.632 .
$$

Therefore, the relaxation times can easily be determined by reading the corresponding time for $Y(t) /$ $Y_{\infty}=0.632$ from the plot. Figure $13 \mathrm{~b}$ shows the assumed case that the total length dilation consists of $70 \%$ Fickian, $20 \%$ of the first relaxational and $10 \%$ of the second relaxational dilation. The data are calculated values. The solid lines point out the fact that the three regimes can be easily distinguished if a logarithmic instead of linear time scale is chosen. In fact, the only reason for the logarithmic time scale is to visualize and identify the different regimes and to simplify the determination of the relaxation times. In Figure 14, this graphical procedure is applied to the data plotted in Figure 12.

The representation of the data by two relaxational functions is sufficient and accurate. This has been verified by fitting experimental data for a number of different experiments numerically. Differences between fitted and graphically obtained relaxation times are smaller than $10 \%$. Differences for the dilation fractions are smaller than $5 \%$. Therefore, a tedious numerical fit procedure for all experiments is not necessary. The determination of the model parameters from the graphs is sufficiently accurate, but quicker.

We have carried out various consecutive integral dilation experiments with the polyimide 6FDAMDA. The first pressure step was from 0 to 3.5 bar.
In each of the following experiments the pressure was increased from 0 to 20.5 bars. The pressure was hold for $80,000 \mathrm{~s}$ at this pressure. The experimentation time in these experiments is much longer compared to experiments with consecutive pressure steps and dilation reaches equilibrium within this time. After each dilation experiment, the pressure vessel was evacuated for $10,000 \mathrm{~s}$ and a new experiment was started. After the third experiment at 20.5 bar, the sample was stored for 30 days at ambient conditions. Then, a new dilation experiment at 20.5 bar was carried out again. The pressure-time profile of this sequence of experiments is shown in Figure

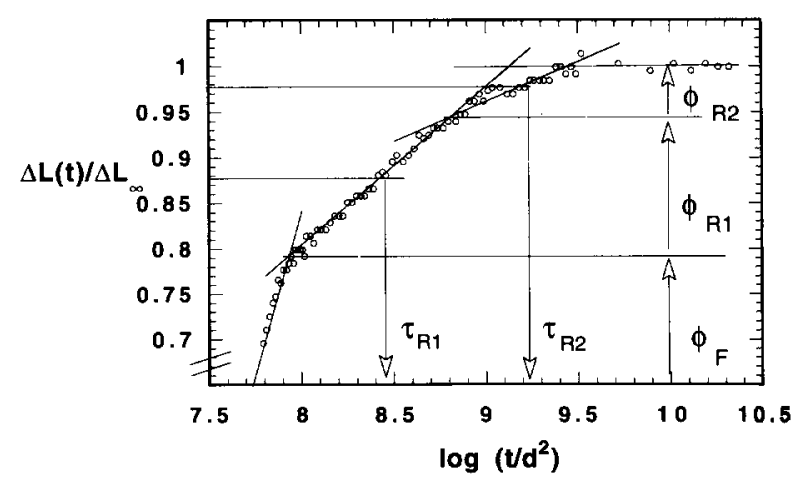

Figure 14. Graphical identification and quantification of different regimes in the experimental dilation kinetics for the sorption of $\mathrm{CO}_{2}$ in $6 \mathrm{FDA}-\mathrm{MDA}$ at $T=35^{\circ} \mathrm{C}$ for an integral pressure step from $p=0$ bar to $p=20$ bar; relaxation times can be determined according to the procedure described in the text. 


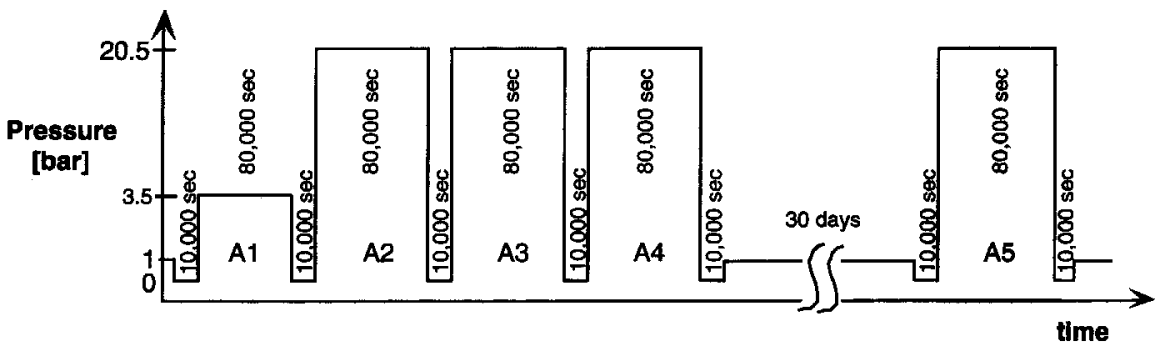

Figure 15. Schematic representation showing the pressure history of the polymer sample.

15. The dilation kinetics of the first two experiments at 20.5 bar (A2 and A3) are shown in Figure 16.

The dilation kinetics measured for the experiment $A 3$ is very different from the kinetics measured in experiment A2. The fraction of Fickian dilation is much larger in experiment A3. Since the absolute dilation stays constant for the two experiments, the relaxational dilation fraction in experiment $\mathrm{A} 3$ is smaller than in experiment A2. The fit parameters for the experiments A2 and A3 and two additional experiments A4 and A5 are summarized in Table 2. The third experiment at 20.5 bar does not show any further increase in $\phi_{F}$ (A4). Leaving the sample for 30 days at ambient conditions results in a considerable decrease of $\phi_{F}$ (A5), but with a value for $\phi_{F}$ still larger than for the second experiment (A2). Low pressure experiments with 6FDA-MDA (A1) show already relaxational dilation in contradiction to the dilation of 6FDA-DAF. Comparing experiments A1 and $A 2$, it is also clear that the Fickian dilation fraction is larger at lower pressures. In all the experiments A1 to A5, the Fickian dilation fraction $\phi_{F}$ shows a very pronounced influence of the swelling history.

Table II also gives the maximum relative dilation. For the sequence of experiments, the maximum di-

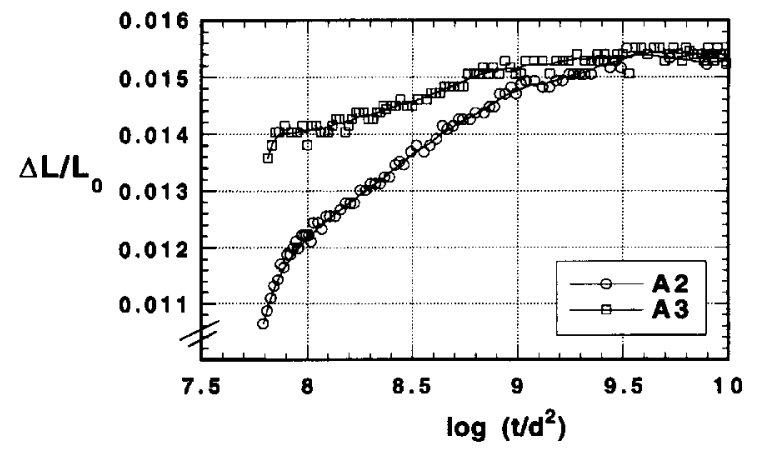

Figure 16. Direct comparison of the dilation kinetics for two consecutive runs (A2 and A3) for 6FDA-MDA at $p=20$ bar and $T=35^{\circ} \mathrm{C}$; see Figure $13 \mathrm{a}$ for further experimental details. lation increases from one experiment to another. The third and fourth experiment were carried out 30 days apart from each other and one may argue that the polymer sample may have consolidated during this time. A new dilation experiment (A5) results in a maximum relative dilation smaller than actually expected for one immediately determined after experiment $\mathrm{A} 4$.

Relaxation times, determined numerically or by the graphical procedure, are not affected by the pressure, i.e., penetrant concentration. The relaxation times are typically about $\tau_{R 1}=2.5 * 10^{8} \mathrm{~s} / \mathrm{cm}^{2}$ for the first relaxation time $\tau_{R 1}$ and $2.5 * 10^{9} \mathrm{~s} / \mathrm{cm}^{2}$ for the second relaxation time $\tau_{R 2}$. This may have its origin in the fact that the relaxation times are empirical in nature and cannot be related to any specific molecular motion. If, however, the relaxation times represent molecular motions, the fitted relaxation time can represent a whole spectrum of molecular motions. Experiments determining polymer chain dynamics, such as NMR with labeled parts of the chain may be more viable to answer the question whether the relaxation dynamics depends on the degree of penetrant-induced stress on the polymer network.

After the experiments with integral pressure steps, the polymer sample was kept for about 35 days at ambient conditions. Then, the sample was used for consecutive pressure steps of 3 bars. Within the experimentation time of $10,000 \mathrm{~s}$ at each pressure, dilation reached equilibrium only for pressures

Table II. Fitting Parameters for Dilation Experiments With Large, Incremental Pressure Steps

\begin{tabular}{cllll}
\hline Experiment & \multicolumn{1}{c}{$\phi_{F}$} & \multicolumn{1}{c}{$\phi_{R 1}$} & \multicolumn{1}{c}{$\phi_{R 2}$} & $\Delta L_{\text {max }} / L_{0}$ \\
\hline A1 & 0.79 & 0.1 & 0.11 & 0.004 \\
A2 & 0.789 & 0.144 & 0.067 & 0.0153 \\
A3 & 0.912 & 0.071 & 0.017 & 0.0154 \\
A4 & 0.915 & 0.068 & 0.017 & 0.0158 \\
A5 & 0.83 & 0.13 & 0.04 & 0.0158 \\
\hline
\end{tabular}


lower or equal than 18.5 bar. The parameter for the analysis are summarized in Table III. From this table, one can conclude that the fraction of Fickian dilation decreases with increasing pressure whereas the fraction of relaxational dilation increases.

\section{Summary of High-Pressure Dilation Experiments}

The experiments carried out in large, incremental pressure steps can be summarized as follows:

1. Distinct regions of Fickian and relaxational dilation were identified

2. The Fickian and relaxational dilation fractions show a pronounced influence of swelling history

3. The maximum relative dilation increases when various dilation experiments are carried out consecutively at the same pressure

4. The relaxation times determined from the dilation kinetics do not depend on the penetrant concentration

The experiments carried out in consecutive, small incremental pressure steps can be summarized as follows:

1. A considerable hysteresis behavior can be observed between dilation and consolidation isotherms. Consolidation isotherms lie always above dilation isotherms.

2. The fraction of Fickian dilation decreases with increasing pressure and the fraction of relaxational dilation increases.

3. No distinct time-dependent regions can be observed in the consolidation kinetics; instead, the curves are smooth.

4. The consolidation kinetics at higher penetrant concentration is faster compared to that at low penetrant concentrations.

5. The amount of residual dilation after dilation experiments at high concentrations depends strongly on the time of evacuation after the experiment. The time scale for these relaxational consolidation processes at "zero" penetrant concentration is much larger than the time scale for the consolidation due to desorption of the penetrant molecules at higher pressures.

In the following paragraph, we will develop a qualitative model that explains the experimental phenomena observed so far.
Table III. Fitting Parameters for Dilation Experiments With Consecutive, Small Incremental Pressure Steps

\begin{tabular}{rllll}
\hline $\begin{array}{c}\text { Pressure } \\
\text { (bar) }\end{array}$ & $\phi_{F}$ & $\phi_{R 1}$ & $\phi_{R 2}$ & $\Delta L_{\text {max }} / L_{0}$ \\
\hline 3.5 & 0.8 & 0.17 & 0.03 & 0.004 \\
6.5 & 0.59 & 0.27 & 0.14 & 0.0064 \\
9.5 & 0.48 & 0.25 & 0.27 & $\mathbf{0 . 0 0 8 6}$ \\
12.5 & 0.44 & 0.28 & 0.28 & $\mathbf{0 . 0 1}$ \\
15.5 & 0.44 & 0.28 & 0.28 & $\mathbf{0 . 0 1 2}$ \\
18.5 & 0.38 & 0.28 & 0.34 & $\mathbf{0 . 0 1 4}$ \\
\hline
\end{tabular}

\section{QUALITATIVE MODEL FOR THE DESCRIPTION OF RELAXATIONAL DILATION PHENOMENA}

Let us assume that the free volume of a glassy polymer matrix can be approximated by a free-volume distribution ${ }^{23,36}$ and that penetrant molecules need a certain energy $E_{i}$ to nest in certain sites in the polymer matrix. At low gas or vapor activity only those places can be occupied which require a low energy. These loci are easy to dilate or they even exist in the glassy matrix. The larger swelling becomes, due to high sorption values, the more energy will be required to strain the polymer network and to occupy a new position in the matrix. This picture can be supported by sorption experiments at different temperatures. Koros et al ${ }^{37}$ measured the sorption isotherms of $\mathrm{CO}_{2}$ in poly (ethylene terephthalate) over a wide range of temperatures. For different temperatures the pressure belonging to a fixed concentration was determined. The slope of a plot of the logarithm of the pressure versus the inverse temperature at a fixed penetrant concentration (Van't Hoff plot) is directly related to the isosteric heat of sorption. Different Van't Hoff plots at different concentration gives the concentration dependence of the isosteric heat of sorption. The fact that the negative isosteric heat of sorption increases with increasing penetrant concentration supports the picture of increasing energy required to strain the polymer network at higher degrees of swelling.

In a sorption experiment at a pressure $p_{1}$ penetrant molecules occupy all loci $n_{i}$ which require an energy $E_{i} \leq E\left(p_{1}\right)$. Upon desorption the number of loci $n_{i}$ and the energy level to occupy them does not change for a second sorption cycle. When the pressure is increased from $p_{1}$ to $p_{2}$, molecules will occupy new loci $n_{j}$ which require energies $E_{j}<E\left(p_{2}\right)$. This process may also be reversible if no morphological alterations in the polymer matrix occur. This was 
experimentally verified by consecutive dilation experiments at a constant pressure of $3 \mathrm{bar}$. Consecutive dilation experiments carried out with the polyimide 6FDA-DAF from 0 to 3 bar are reproducible. There will be, however, a penetrant concentration from which on the penetrant induced stresses on the polymer matrix are of such a magnitude that the morphology will not remain unaffected and relaxational dilation will occur. This also can be the case at a low concentration as shown for the polyimide $6 \mathrm{FDA}$ MDA for a pressure step from 0 to 3.5 bar.

We now assume that these morphological alterations find their expression in a decrease of the energy levels required to occupy certain positions. Or in other words, if $n\left(E_{2}\right)$ is the number of molecules directly sorbed at the energy level $E\left(p_{2}\right)$ in the virgin polymer, then the morphology alterations result in an increase of available loci $n_{\text {total }}$ at this energy level. The amount of loci $n\left(E_{2}\right)$ originally available in the virgin polymer will be called "Fickian" $n_{F}$, whereas the newly created number will be called "relaxational" $n_{R}$.

$$
n_{\text {total }}=n_{F}+n_{R}
$$

Since the morphological alterations and therefore the creation of new sites $n_{R}$ are slow in nature, the ordinary Fickian sorption and the relaxational sorption are easy to distinguish.

If all penetrant molecules are now desorbed by applying a vacuum, the polymer remains with more sorption sites belonging to the pressure level $p_{2}$ than the virgin one. In a second experiment briefly after the first sorption experiment, the relaxational sites will be the new "Fickian" sorption sites. The new Fickian dilation fraction will be the sum of the old Fickian sorption sites $\phi_{F}$ plus relaxational sites $\phi_{R}$ stemming from the first experiment. Depending on the time between the first sorption experiment and a second one at the same applied pressure, the sorption and hence the dilation kinetics may be very different. If the second experiment were carried out at a considerably later time, for instance one month later, the former relaxational sites (new Fickian sites) consolidate into the direction of a new equilibrium and the number of Fickian sites observed will be considerably smaller than in an experiment immediately carried out after the first experiment.

To observe these phenomena, one condition must be fulfilled: the relaxation process for the creation of new Fickian sites must be considerably faster than the consolidation relaxation. Actually, this hypothesis is easy to accept if one accepts the fact that molecular motions of polymer chains are faster in the presence of solvent or plasticizer molecules. This "lubrication" effect ${ }^{38}$ of $\mathrm{CO}_{2}$ molecules on polymer chains is only present during the sorption experiment but not during desorption experiments at vacuum. It is clearly demonstrated by the following experiment: a polymer strip of uncross-linked Nitril butylrubber $(\mathrm{NBR})(10 \mathrm{~cm} * 1 \mathrm{~cm} * 0.1 \mathrm{~cm})$ was placed in the dilation setup at $p=10$ bar $\mathrm{CO}_{2}$. After three days the strip was about twice as long due to the creep induced by its own weight. Another polymer strip of the same polymer hanging in ambient atmosphere did not extend more than $10 \%$ in length within 2 months. The $\mathrm{CO}_{2}$ had softened the polymer and enhanced the polymer chain motions. With this in mind it can be understood that the sorption relaxation is faster than the consolidation relaxation.

Until now, only experiments with integral pressure steps have been considered where the penetrant concentration in the polymer film changes from concentration $c=0$ at $t=0$ to an equilibrium concentration $c=c_{\text {equil }}$ at $t=\infty$. Now we consider the situation where an equilibrium concentration $c_{1, \text { equil }}$ is already present in the polymer when the next pressure step (from $p_{1}$ to $p_{2}$ ) is applied. Following the argumentation in the first part of this section, a part of the sites for the pressure step from $p_{1}$ to $p_{2}$ has been occupied in the pressure step from 0 to $p_{1}$ due to polymer matrix relaxation. These sites were "Fickian" sorption sites for the pressure step from $p_{1}$ to $p_{2}$. But since they are already occupied, the sites cannot contribute to the Fickian dilation fraction and $\phi_{F}\left(p_{1} \rightarrow p_{2}\right)$ in the case of polymer matrix relaxation is smaller than in the ideal case of elastic reversible dilation. As a consequence, the ratio of relaxational sites to "Fickian" sites increases. Successive pressure steps result in a decreasing "Fickian" sorption site fraction with increasing fraction of relaxational sites. The arrow diagrams in Figure $17 \mathrm{a}$ and $\mathrm{b}$ compare the situation (a) without any relaxational sorption with (b) the situation of relaxational sorption. From this figure it can be seen that the Fickian part of the dilation kinetics decreases with incrementally increasing pressure steps as described above. The situation may occur where no Fickian dilation can be observed from a certain pressure on.

The proposed model can also account for the consolidation process and here especially for the hysteresis behavior shown in Figure 11. The arrow diagram in Figure 18 visualizes the argumentation. It has been shown that relaxational sites can act as Fickian sites in a second run briefly after the first one. This is based on the assumption that no energetic difference exists between these two kinds of 


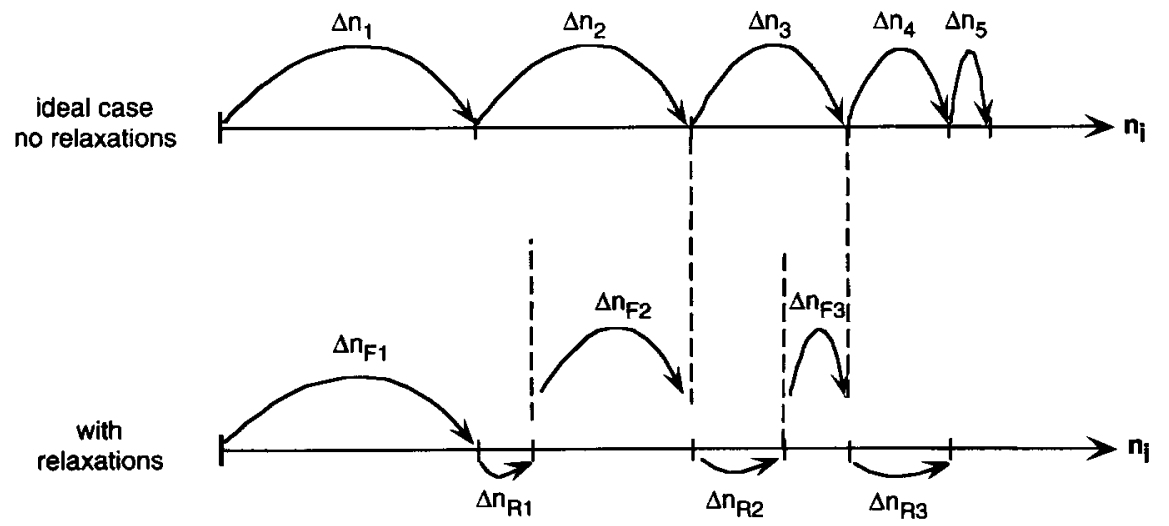

Figure 17. Visualization of the phenomenon of disappearance of the Fickian dilation part in dilation kinetics for incrementally increasing pressure steps.

sorption sites. This, of course, is also true for the molecules leaving the polymer in a desorption step between the two successive sorption runs. The question, which molecules will leave the polymer matrix first, the Fickian or relaxational ones, is therefore inadequate. The question to be asked is: which sites, the relaxational or Fickian sites, will consolidate faster? On a molecular scale, we anticipate Fickian dilation to be related to motions of short parts of the polymer chain; relaxational dilation is related to motions of long polymer chain segments. Since fewer polymer segments contribute to the short chain motion, Fickian consolidation is much faster than relaxational consolidation. The relaxational dilation remains in the polymer causing the consolidation isotherm to lie above the dilation isotherm.

During the consolidation process, the penetrant molecules may, however, interchange between former Fickian and relaxational sites because these are energetically equally easy to occupy. At low pressures coming from elevated pressures, these quick consolidation processes coincide with slow relaxational processes stemming from a pressure decrease step at a higher pressure level. The rate constants for these relaxation processes can not be distinguished from quick processes and the consolidation kinetics shows no distinct regions. Instead, the consolidation kinetics has a smooth shape as it was shown in Figure 10. Due to the smaller lubrication effect for a negative pressure step compared to the positive pressure step in dilation, the consolidation relaxations will be even slower than the dilation relaxations. Consolidation relaxations can be so slow on the experimental time scale that residual dilation can be observed. The residual dilation also decreases in time, but it is unlikely that the polymer sample consolidates into its original volume.

\section{CONCLUSIONS}

This study describes in detail the development of an automated experimental method to determine the
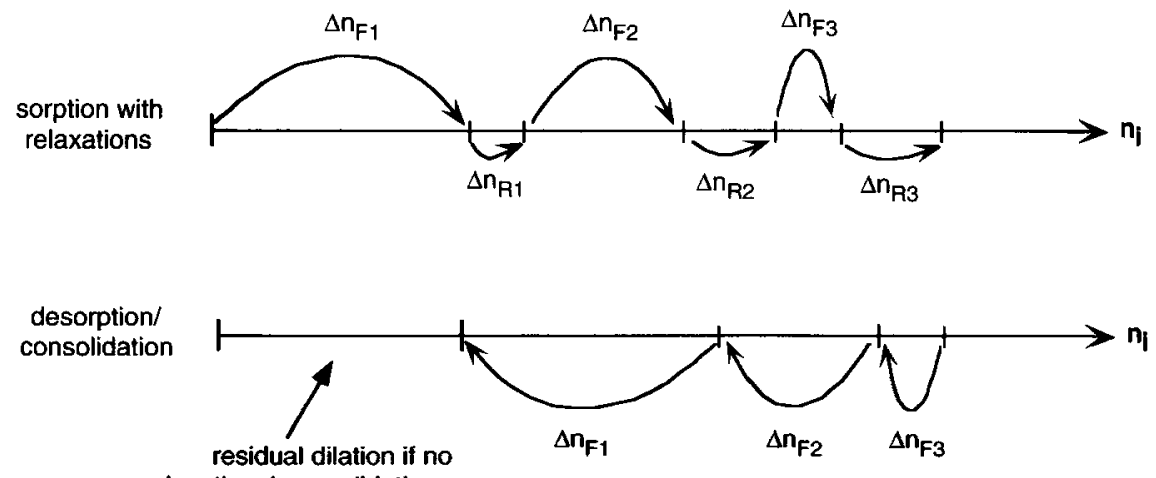

relaxational consolidation occurs

Figure 18. Visualization of the hysteresis phenomenon between the dilation and the consolidation isotherm. 
sorption-induced dilation kinetics of a polymer/ penetrant system. Systematic dilation experiments were carried out with carbon dioxide and two aromatic polyimides. Experiments carried out with the polyimide 6FDA-DAF at low pressures lead to the conclusion that the measured dilation kinetics can be related to the sorption kinetics. The polymer sample dilates simultaneously with the concentration increase in the center of the polymer strip.

Experiments at high carbon dioxide pressures up to 36 bar were carried out in two different ways: (a) large, integral pressure steps and (b) small, consecutive incremental pressure steps. In both kinds of experiments, fast and slow dilation kinetics can be distinguished. Integral pressure step experiments show a pronounced effect of the swelling behavior on the sample history. Incremental pressure step experiments show a significant difference between dilation and consolidation. We propose a model that distinguishes fast, Fickian dilation and slow, relaxational dilation. The model explains qualitatively the experimental observations.

\section{REFERENCES AND NOTES}

1. D. R. Paul and Yu.P. Yampol'skii, eds., Polymeric Gas Separation Membranes, CRC Press Inc., Boca Raton, 1994.

2. W. J. Koros, Barrier Polymers and Structures, ACS Symp. Ser. 1990, p. 423.

3. N. Toshima, ed., Polymers for Gas Separation, VCH Publishers Inc., New York, 1992.

4. E. Smit, Ph.D. thesis, Enschede/The Netherlands, 1991.

5. E. Hensema, Ph.D. thesis, Enschede/The Netherlands, 1991.

6. M. Wessling, v.d., Th. Boomgaard, M. H. V. Mulder, and C. A. Smolders, Makromol. Chem., Macromol. Symp., 70/71, 379 (1993).

7. P. J. Flory, Principles of Polymer Chemistry, Cornell University Press, Ithaca, NY, 1953.

8. R. M. Barrer, A. J. Barrie, and J. Slater, J. Polym. Sci., 27, 177 (1958).

9. A. S. Michaels, W. R. Vieth, and J. A. Barrie, J. Appl. Phys., 34, 1 (1963).

10. W. R. Vieth, H. H. Alcalay, and A. J. Frabetti, J. Appl. Polym. Sci., 30, 1261 (1964).

11. D. Raucher and M. Sefcik, in Industrial Gas Separation, ACS Symp. Ser., 111, 223 (1983).

12. J. S. Vrentas and C. M. Vrentas, Macromolecules, 24, 2404 (1991).
13. J. G. A. Bitter, Transport Mechanisms in Membrane Separation Processes, Plenum Press, New York, 1991.

14. G. G. Lipscomb, AIChE J., 36, 1505 (1990).

15. T. A. Barbari and R. M. Conforti, J. Polym. Sci., Polym. Phys. Ed., 30, 1261 (1992).

16. G. H. Weiss, J. T. Bender, and M. F. Shlesinger, Macromolecules, 25, 990 (1992).

17. R. Kirchheim, Macromolecules, 25, 6952 (1992).

18. H. B. Hopfenberg, J. Membrane Sci., 3, 215 (1978).

19. D. S. Pope, G. K. Fleming, and W. J. Koros, Macromolecules, 23, 2988 (1990).

20. A. C. Puleo and D. R. Paul, J. Membrane Sci., 47, 301 (1989).

21. A. C. Puleo, N. Muruganadam, and D. R. Paul, $J$. Polym. Sci., Polym. Phys. Ed., 27, 2385 (1989).

22. H. C. Park, Ph.D. thesis, University of Twente, The Netherlands, 1993.

23. M. Wessling, Ph.D. thesis, University of Twente, The Netherlands, 1993.

24. G. R. Husk, C. E. Cassidy, and K. L. Gebert, Macromolecules, 21, 1234 (1988).

25. K. Tanaka, M. Okano, H. Toshino, H. Kita, and K. Okamoto, J. Polym. Sci., Polym. Phys. Ed., 30, 907 (1992).

26. D. S. Pope, W. J. Koros, and G. K. Fleming, J. Polym. Sci., Polym. Phys. Ed., 27, 1173 (1989).

27. Y. Kamiya, T. Hirose, and Y. Naito, J. Polym. Sci., Polym. Phys. Ed., 24, 2107 (1986).

28. W. J. Koros and D. R. Paul, J. Polym. Sci., Polym. Phys. Ed., 14, 1903 (1976).

29. M. Wessling, S. Schoeman, v.d., Th. Boomgaard, and C. A. Smolders, Gas Sep. \& Purif., 5, 222 (1991).

30. G. K. Fleming and W. J. Koros, Macromolecules, 19, 2285 ( 1986 ).

31. N. L. Thomas and A. H. Windle, Polymer, 22, 627 (1981).

32. J. Crank, The Mathematics of Diffusion, 2nd ed., Clarendon Press, Oxford, 1975.

33 J. Crank and G. S. Park, Diffusion in Polymers, Academic Press, New York, 1968.

34. Y. Kamiya, T. Hirose, K. Mizoguchi, and K. Terada, J. Polym. Sci., Polym. Phys. Ed., 26, 1409 (1988).

35. A. R. Berens and H. B. Hopfenberg, Polymer, 19, 489 (1978).

36. V. M. Shah, S. A. Stern, and P. J. Ludovice, Macromolecules, 22, 4662 (1989).

37. W. J. Koros, D. R. Paul, and G. S. Huvard, Polymer, 20, 956 (1979).

38. J. K. Sears and N. W. Touchettes, in Encyclopedia of Polymer Science and Technology, Supplement, Wiley \& Sons, New York, 1989.

Received September 6, 1994

Revised November 30, 1994

Accepted January 10, 1995 